\title{
Formalization of the DE2 Language
}

\author{
Warren A. Hunt Jr. and Erik Reeber \\ Department of Computer Sciences, \\ 1 University Station, M/S C0500, \\ The University of Texas, \\ Austin, TX 78712-0233, USA \\ \{hunt, reeber\}@cs.utexas.edu
}

\begin{abstract}
We formalized the DE2 hierarchical, occurrence-oriented finite state machine (FSM) language, and have developed a proof theory allowing the mechanical verification of FSM descriptions. Using the ACL2 functional logic, we have defined a predicate for detecting the wellformedness of DE2 expressions. Furthermore, we have defined a symbolic simulator for DE2 expressions which also serves as a formal cycle-based semantics for the DE2 language. DE2 is deeply embedded within ACL2, and the DE2 language includes an annotation facility that can be used by programs that manipulate DE2 descriptions. The DE2 user may also specify and prove the correctness of programs that generate DE2 descriptions. We have used DE2 to mechanically verify components of the TRIPS microprocessor implementation.
\end{abstract}

\section{Introduction}

We present a formal description of and proof mechanism for the DE2 hierarchical, occurrence-oriented finite state machine (FSM) description language, which we use to design and verify FSM-based designs or to optimize existing designs in a provably correct manner. This definition is primarily aimed at the representation and verification of hardware circuits, but DE2 could also be used in other areas such as protocols and software processes. Defining a hardware description language (HDL) is difficult because of the many different ways in which it may be used; for example, a HDL may be used to specify a simulation semantics, define what circuits can be specified, restrict allowable names, enforce circuit interconnect types, estimate power consumption, and provide layout or other manufacturing information. We have formally described the DE2 language using the ACL2 logic [16], and we have formally verified DE2 descriptions using the ACL2 theorem prover.

DE2 is designed to permit the rigorous hierarchical description and hierarchical verification of finite-state machines (FSMs). We call our language DE2 (Dual-Eval 2) because of the two-pass approach that we employ for the language recognizers and evaluators. DE2 is actually a general-purpose language for specifying FSMs; users may define their own language primitives. We recognize valid DE2 descriptions with an ACL2 predicate that defines the permissible syntax,

D. Borrione and W. Paul (Eds.): CHARME 2005, LNCS 3725, pp. $20-342005$.

(C) IFIP International Federation for Information Processing 2005 
names, and hierarchy, of valid descriptions. The DE2 language also provides a rich annotation language that can be used to enforce syntactic and semantic design restrictions.

We begin our presentation by listing DE2 language characteristics, contrasting the DE2 language with other related efforts, and presenting some DE2 language examples. We next present the definition of DE2's simulation-based semantics. We conclude by describing how we use the DE2 language to verify circuits from the TRIPS microprocessor design [7.

\section{DE2 Language Features}

The development of DE2 required balancing many demands. In particular, the demand for hardware verification requires that it be as simple as possible to evaluate, translate, and extend. In this section we list the resulting characteristics of DE2.

- Hierarchical: A module is defined by connecting submodules.

- Occurrence-Oriented: Each reference to a previously defined module is called an occurrence. All named modules are defined as a sequence of occurrences (unnamed lambda modules are discussed in Section 4.2).

- Deep Embedding in ACL2: DE2 modules are represented as ACL2 constants. Using the terminology defined by Boulton et al. 13, DE2 is deeply embedded in the ACL2 language. This embedding allows us to write ACL2 functions which simulate, analyze, generate, and manipulate DE2 modules.

- Annotation Mechanisms: We use annotations to describe elements of a circuit which are not defined by evaluation (e.g. layout information). In DE2, annotations are first class objects.

- Parameterized Finite Types: In DE2, every module input and output is a bit vector of parameterized length. When the lengths of all the inputs and outputs are known, we may appeal to BDD- and SAT-based techniques for verification.

- Two-pass Evaluation: A DE2 module is evaluated by twice interpreting its list of occurrences. This two-pass evaluation necessitates a level-order for the combination functions.

- Representation of Internal State: We represent the internal state of a module as an arbitrary block of memory that is implicitly part of the module's input and output and is updated during the second evaluation pass. This representation limits us to designing FSMs, but greatly simplifies the design and verification of these machines.

- User-defined Primitive Modules: We allow users to define primitive modules, rather than requiring that primitive modules be built into the language.

- User-selectable Libraries: Sets of primitives can become libraries. Libraries can be loaded into similar projects, which allows reuse of modules and verification efforts. 
- Verified DE2 Language Generators: We can write ACL2 functions which generate DE2 modules. Since the semantics of DE2 have been formalized in ACL2, these functions can be shown to always generate correct DE2 code.

- Hierarchical Verification: Our verification process involves verifying properties of submodules and then using these properties to verify larger modules built from these submodules. This hierarchical technique allows us to avoid reasoning about the internals of complex submodules.

- Books for Verification Support: We have defined a number of ACL2 "books" to assist the verification of DE2 modules. When loaded into the theorem prover, these books use the ACL2 semantics of DE2 to verify properties of DE2 modules. We have used these books on a number of verification projects, some of which involve the verification of ACL2 functions that generate DE2 circuits.

\section{Related Work}

The hardware verification community has taken two approaches [13] to defining the semantics of circuits: shallow and deep embedding. Shallow embedding defines a circuit description as a first-class object in a well-defined subset of a formal language. The syntax and formal semantics of the HDL are therefore a subset of the semantics of the formal language. Deep embedding defines a circuit description as a constant in a formal language. The syntax and semantics of the HDL are then written in the formal language.

The DE2 language has been defined by deeply embedding it inside the ACL2 language, a primitive recursive functional subset of Lisp [17]. By embedding DE2 within ACL2, we are given access to a theorem proving environment which has successfully verified large-scale hardware systems 89. The formalization of the DE2 language is similar in style to the embedding of the DUAL-EVAL HDL in NQTHM [11] and the DE language in ACL2 [10]. The DE language is different from DUAL-EVAL in that DE permits user-defined primitives, re-usable libraries, annotations, and contains a different structuring of data for stateholding elements. The DE2 language contains the new features of DE, but also has a parameterized type system, a more sophisticated system for applying non user-defined primitives (implemented as ACL2 functions), and a more automated verification system.

In other hardware verification efforts with ACL2, hardware descriptions were translated directly to ACL2 models in the style of shallow-embedding [89]. These efforts do not permit the syntactic analysis of the circuits so represented; that is, it is not possible to treat the circuit descriptions as data so a program may be used to analyze its suitability.

Tom Melham used the HOL system [12 to deeply embed some elements of a hardware description language [12. Boyer and Hunt attempted to deeply embed a subset of VHDL in the ACL2 logic, but this specification grew to more than 100 pages of formal mathematics, and its usefulness became suspect. Deeply 
embedding a HDL into another language brings great analytical power at the cost of having to manage all of the logical formalisms required-but these formalisms represent the real complexity that are inherit in such languages and in their associated analysis and simulation systems. To make such an embedding useful, a serious effort needs to be made to ensure an absolute economy of complexity, and there needs to be libraries that ease the use of such an embedding.

A significant amount of work has focused on the use of functional programming languages to simplify the writing of HDL-based descriptions. Mary Sheeran has developed the language Lava [1] and she has used it to design fast multipliers [2]. The strengths of Lava is its facilities to write programs that generate hardware - similar to the ACL2 programs we write to generate DE2 descriptions - and its ability to embed layout information in the Lava language - similar to annotations in DE2. The Lava implementation does not include an associated reasoning system, but a user can appeal to SAT procedures to compare one Lava description with another.

Our recent verification methodology, which combines a SAT-based decision procedure with theorem proving, was partially inspired by the work at Intel combining symbolic trajectory evaluation with theorem proving. This work makes use of the functional languages Lifted-FL [4] and reFLect [3. Some of the ways DE2 differs from these languages include its simpler semantics (e.g. two pass evaluation), its simple syntax, its close correspondence to a subset of Verilog, and its embedding within a general-purpose theorem prover.

\section{Example}

The use of the DE2 language is similar to the use of other hardware description languages. Circuits are specified in a hierarchical manner, and the syntactic form of the hierarchical circuit description also defines the hierarchical structure of a description's associated state. Here we give an example of a DE2 circuit specification, and describe some of the restrictions imposed by the DE2 language.

Our DE2 language definition is a tremendous abstraction of the physical reality. The DE2 language defines finite-state machines by permitting a user to define primitive elements. For this section, we assume the definition of Boolean connectives and state-holding elements have already been given. Issues such as clocking, wire delay, race conditions, power distribution, and heat, have been largely ignored.

Informally, the DE2 language hierarchically defines Mealy machines - i.e. the outputs and next state of every module is a function of its inputs and internal state. By successively repeating the evaluation of an identified FSM, the DE2 system can be used to emulate typical finite-state machine operation. DE2 language definitions obey the syntax of Lisp constant expressions; that is, module definitions are represented as Lisp data rather than Lisp function definitions, macros, or other such constructs. We first give an example of several combinational circuits, where we exhibit some of the restrictions our evaluation approach imposes. Later we exhibit a sequential circuit. 


\subsection{Combinational Modules}

Consider the circuit shown in Figure 1. In DE2, this circuit is represented as follows.

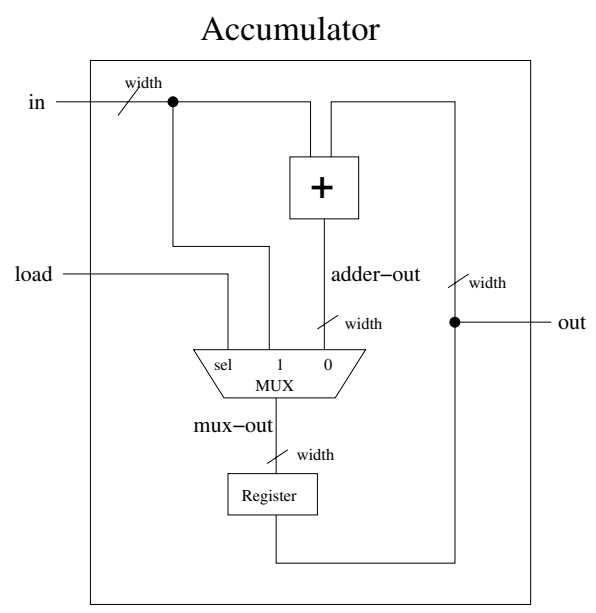

Fig. 1. Schematic of an Accumulator

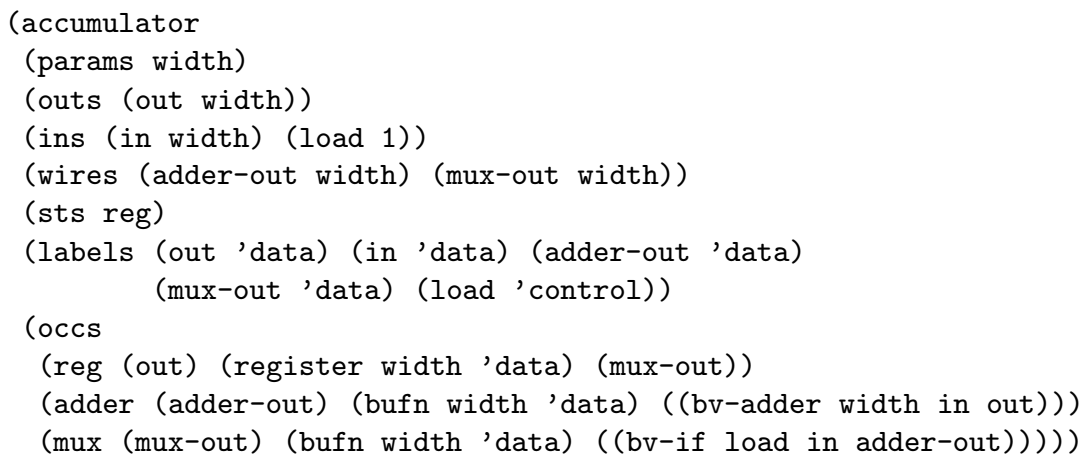

A module is identified by its name, in this case accumulator. Each module is composed of a set of key-value pairs whose entries depend on the type of the module. All modules have lists of parameters, outputs, inputs, and states identified by params, ins, sts, and outs, respectively. Modules can also have a list of wires local to the module, identified by wires. This module also has a labels entry, which is an annotation. Annotations are not required, but can be used to enable optimizations, assist verification, or provide information to other tools. In this case, we use the labels annotation, along with a static checker, to ensure that we do not use a data wire when a control wire was expected or vice versa. Annotations can also be used to represent layout information or other physical attributes - a user may define their own annotations. 
A module will also include occurrences which define the relationship between its inputs, outputs, and internal modules. Each occurrence consists of a unique occurrence name, a list of outputs, a module reference combined with its parameter list, and a list of inputs. For example, the first occurrence in the above example is named reg. The reg occurrence consists of an instance of a register module with the parameter width, input mux-out, and output out. The fact that reg occurs in the accumulator module's sts list denotes that it is a state-holding occurrence. Each input to an occurrence is specified by an ACL2 expression of the inputs and internal "wires" of the module. Our primitive simulation-based evaluator only defines a finite list of ACL2 functions (e.g. bv-adder and bv-if) for use in such an expression.

The DE2 language evaluation semantics define the outputs of a module as a function of its inputs and internal state. The next state of a module is also a function of a module's inputs and internal state. Evaluation is discrete; that is, there is an implicit notion of time which is broken into discrete steps.

Module evaluation begins by binding input values to a module's inputs and binding state values to a module's states. Each occurrence is then evaluated in the order of its appearance. An occurrence is evaluated by binding its inputs and state to the specified arguments and then evaluating the reference itself. For the module defined above, the occurrence reg is evaluated first; the output of a register depends only on its internal state, not its inputs. After the value of mux-out is determined by evaluating the mux occurrence then internal state of the reg occurrence is updated.

In Section 6.1 we present some properties of this example which we have proven mechanically. Using the ACL2 theorem prover, we prove that for any data-path width a LOAD of A (i.e. load is high, in is A) followed by an ADD of $\mathrm{B}$ (i.e. load is low, in is B) produces the addition of $\mathrm{A}$ and $\mathrm{B}$.

\subsection{Primitives}

A primitive module, corresponding to a hardware component built-in to a synthesis tool, has a similar definition to that of a non-primitive module. The difference between a primitive module is that rather than being defined in terms of occurrences of submodules, a primitive module is defined by lisp functions accessed through lambda modules. A lambda module has formals corresponding to the occurrence's list of parameters followed by the occurrence's list of inputs. The lambda module evaluates to a list with its first element being the state of the lambda module followed by its outputs. For example, the following is a definition of the primitive module bufn, which is a submodule of our accumulator.

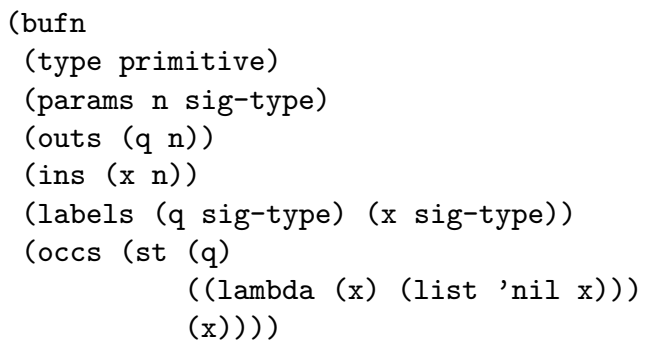


The bufn module instantiates a single lambda module. Since the bufn module has no state, this lambda expression evaluates to a list whose first element is nil. The output of the bufn module, which corresponds to the second element of the list, is equal to its input. The other primitive module found in our accumulator example, register, is defined as follows.

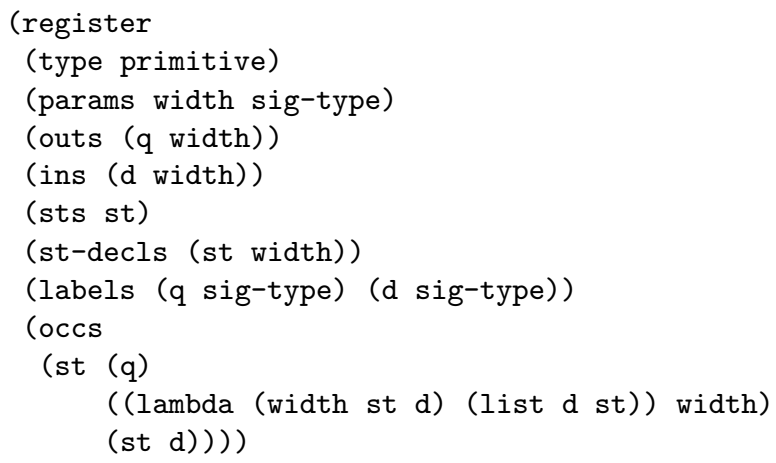

The register example shows how a state-holding primitive is defined in DE2. The state of the register module is accessed through a lambda module named st, which turns the implicit input and output of state into an explicit input and output. The lambda module returns its input $d$ as the next state and its state st as its output. Note that the register module also has a new field, st-decls, that declares the state element st to be a bit-vector of length width. This declaration is not a requirement of DE2 modules, but enables the later use of decision procedures.

\section{The DE2 Evaluator}

The definition of the DE2 evaluator is composed of two groups, each containing two mutually recursive functions. These four functions implement the entire hierarchical evaluation of the outputs and next-state values for any well-formed hierarchical FSM defined using the DE2 language, except for the evaluation of the lambda and ACL2 (primitive) expressions. This set of functions was designed with a number of different goals in mind, so design decisions were made to attempt to implement the desired properties while keeping the size of the system as small as possible.

The DE2 language can be thought of as having two parts: primitive operations and interconnect. We have defined different primitive evaluators, depending on our needs. The primitive evaluator we use for verification of gatelike primitives interprets such primitive modules by applying ordinary Boolean operations. If we are interested in the fan-out of a set of signals, we use a different primitive evaluator. If we want to generate a count of the number of and type of primitive modules required to implement a referenced module, we use a primitive evaluator that collects that information from every primitive encountered during an evaluation pass - note that this does not 
just count the number of defined modules, but it counts the number of every kind of modules required to realize the FSM being evaluated. If we want to compute a crude delay or power estimate, we use other primitive evaluators.

The semantic evaluation of aDE2 design proceeds by binding actual (evaluated) parameters (both inputs and current states) to the formal parameters of the module to be evaluated; this in turn causes the evaluation of each submodule. This process is repeated recursively until a primitive module is encountered, and the specified primitive evaluator is called after binding the necessary arguments. This part of the evaluation can be thought of as performing all of the "wiring"; values are "routed" to appropriate modules and results are collected and passed along to other modules or become primary outputs. This set of definitions is composed of four (two groups of) functions (given below), and these functions contain an argument that permits different primitive evaluators to be used.

The following four functions completely define the evaluation of a netlist of modules, no matter which type of primitive evaluation is specified. The functions presented in this section constitute the entire definition of the simulator for the DE2 language. This definition is small enough to allow us to reason with it mechanically, yet it is rich enough to permit the definition of a variety of evaluators. The se function evaluates a module and returns its primary outputs as a function of its inputs. The de function evaluates a module and returns its next state; this state will be structurally identical to the module's current state, but with updated values. Both se and de have sibling functions, se-occ and de-occ respectively, that iterate through each sub-module referenced in the body of a module definition. We present the se and de evaluator functions to make clear the importance we place on making the definition compact.

The se and de functions both have a flg argument that permits the selection of a specific primitive evaluator. The $f n$ argument identifies the name of a module to evaluate; its definition should be found in the netlist. The ins and st arguments provide the primary inputs and the current state of the fn module. The params argument allows for parameterized modules; that is, it is possible to define modules with wire and state sizes that are determined by this parameter. The env argument permits configuration or test information to be passed deep into the evaluation process.

The se-occ function evaluates each occurrence and returns an environment that includes values for all internal signals. The se function returns a list of outputs by filtering the desired outputs from this environment. To compute the outputs as functions of the inputs, only a single pass is required.

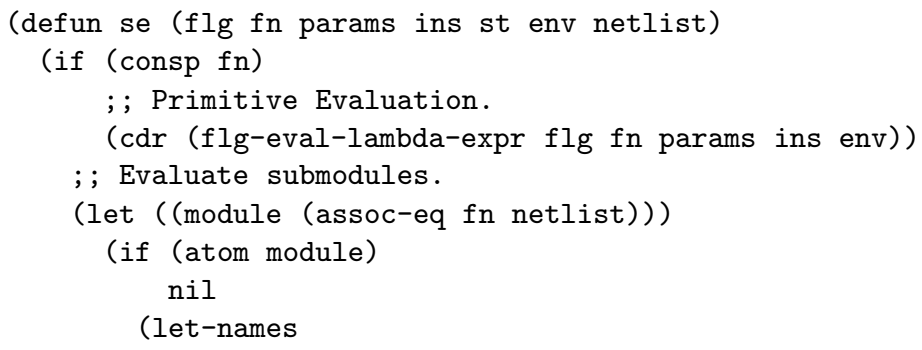




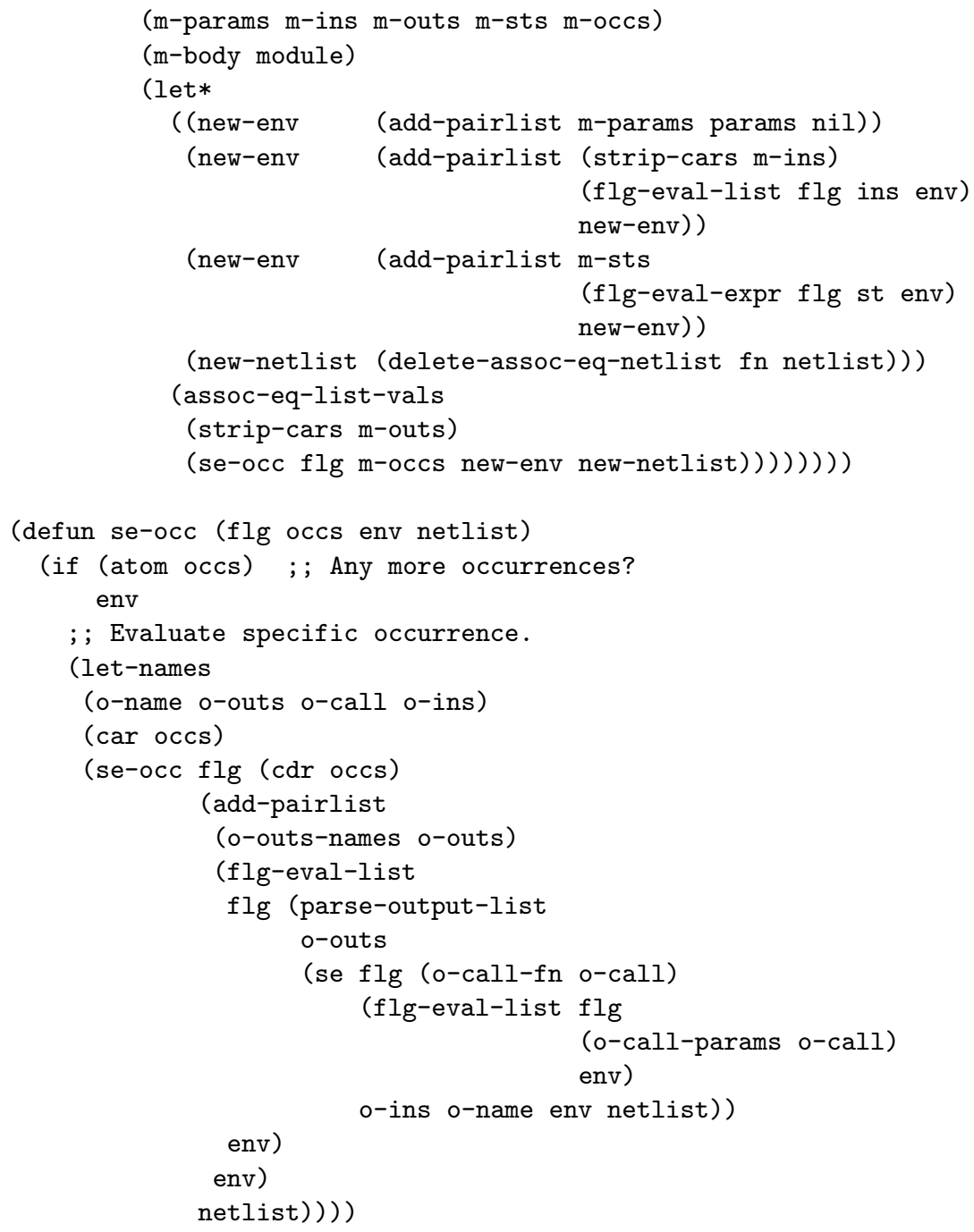

Similarly, the functions de and de-occ perform the next-state computation for a module's evaluation; given values for the primary inputs and a structured state argument, these two functions compute the next state of a specified module. This result state is structured isomorphically to its input's state. Note that the definition of de contains a reference to the function se-occ; this reference computes the value of all internal signals for the module whose next state is being computed. This call to se-occ represents the first of two passes through a module description when DE is computing the next state.

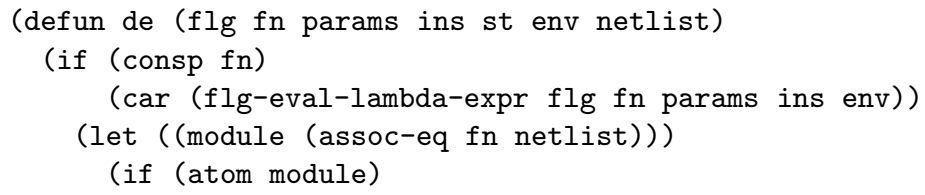




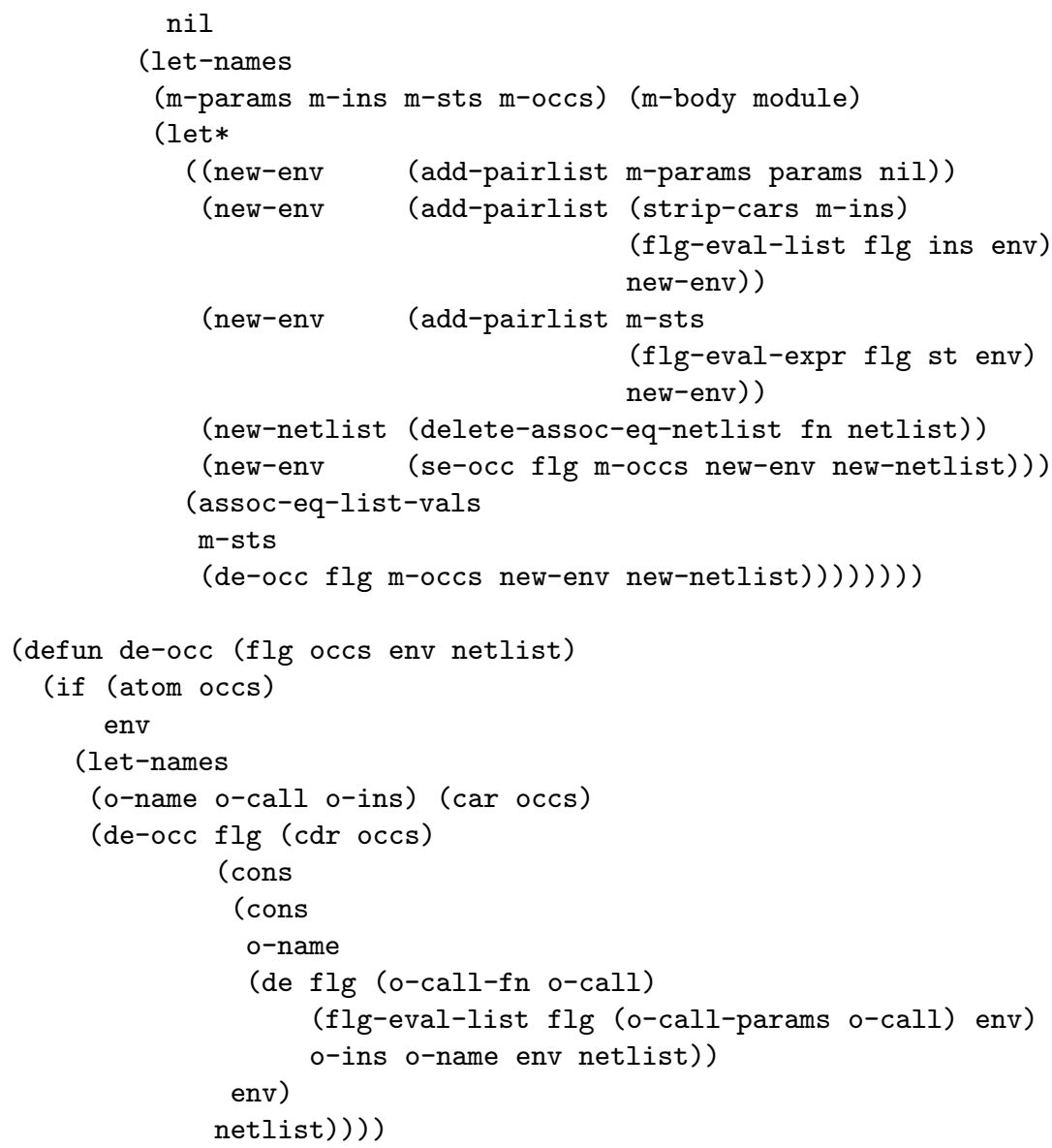

This completes the entire definition of the DE2 evaluation semantics. This clique of functions is used for all different evaluators; the specific kind of evaluation is determined by the $\mathrm{flg}$ input. We have proved a number of lemmas that help to automate the analysis of DE2 modules. These lemmas allow us to hierarchically verify FSMs represented as DE2 modules. We have also defined simple functions that use de and se to simulate a DE2 design through any number of cycles.

An important aspect of this semantics is its brevity. Furthermore, since we specify our semantics in the formal language of the ACL2 theorem prover, we can mechanically and hierarchically verify properties about any system defined using the DE2 language.

\section{Our Use of the DE2 System}

Having an evaluator for DE2 written in ACL2 enables many forms of verification. In Figure 2 we illustrate our verification system, which is built around the DE2 language. 


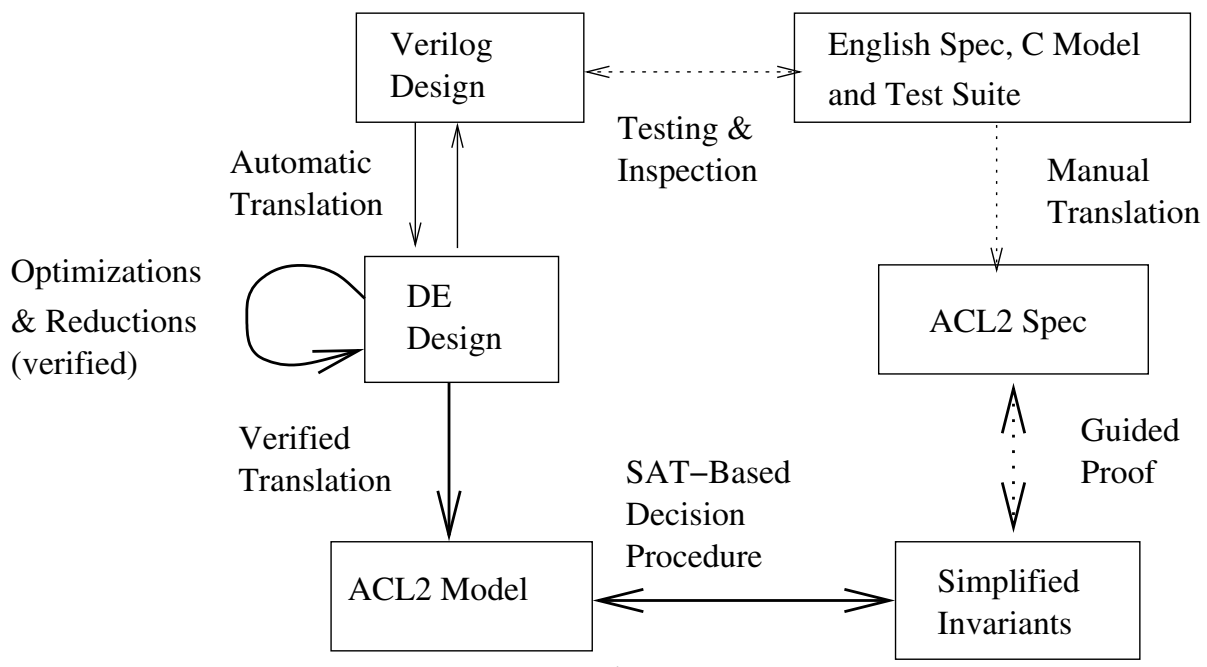

Fig. 2. An overview of the DE2 verification system

We typically use the DE2 verification system to verify Verilog designs. These designs are denoted in the upper left of Figure 2. Currently, the subset of Verilog includes arrays of wires (bit vectors), instantiations of modules, assignment statements, and some basic primitives (e.g. \&, ?: and I). We also allow the instantiation of memory (array) modules and vendor-defined primitives.

We have built a translator that translates a Verilog description into an equivalent DE2 description. Our translator parses the Verilog source text into a Lisp expression, and then an ACL2 program converts this Lisp expression into a DE2 description.

We have also built a translator that converts a DE2 netlist into a cycleaccurate ACL2 model. This translator also provides an ACL2 proof that the DE2 description is equivalent to the mechanical produced ACL2 model. The process of translating a DE2 description into its corresponding ACL2 model includes a partial cone-of-influence reduction; an ACL2 function is created for each module's output and parts of the initial design which are irrelevant to that output are removed. The DE2 to ACL2 translator allows us to enjoy both the advantages of a shallow embedding (e.g. straightforward verification) and the advantages of a deep embedding (e.g. syntax resembling Verilog).

We start with an informal specification of the design in the form of English documents, charts, graphs, C-models, and test code which is represented in the upper right of Figure 2. This information is converted manually into a formal ACL2 specification. Using the ACL2 theorem prover, these specifications are simplified into a number of invariants and equivalence properties. If these properties are simple enough to be proven by our SAT-based decision procedure, we prove them automatically; otherwise, we simplify such conjectures using the ACL2 theorem prover until we can successfully appeal to some automated decision procedure. 
We also use our system to verify sets of DE2 descriptions. This is accomplished by writing ACL2 functions that generate DE2 descriptions, and then proving that these functions always produce circuits that satisfy their ACL2 specifications.

Since DE2 descriptions are represented as ACL2 constants, functions that transform DE2 descriptions can be verified using the ACL2 theorem prover. By converting from Verilog to DE2 and from DE2 to back into Verilog, we can use DE2 as an intermediate language to perform verified optimizations. Another use of this feature involves performing reductions or optimizations on DE2 specifications prior to verification. For example, one can use a decision procedure to determine that two DE2 circuits are equivalent and then use this fact to avoid verifying properties of a less cleanly structured description.

We can also build static analysis tools, such as extended type checkers, in DE2 by using annotations. In DE2, annotations are first-class objects (i.e. annotations are not embedded in comments). Therefore an annotation, such as the labels annotation in Section 4, is parsed as easily as any core language feature. Such static checkers, since they are written in ACL2, can be analyzed and can also assist in the verification of DE2 descriptions. Furthermore, annotations can be used to embed information into a DE2 description to assist with synthesis.

\subsection{Verification Example}

To verify the DE2 circuit in Section 4, we first generate an ACL2 model which is equivalent to the DE2 circuit. The following theorems, which are proven automatically through a proof generated by our translator, prove that the ACL2 functions accumulator-next-st and accumulator-out produce the next state and the out output of the accumulator module from Section 4.

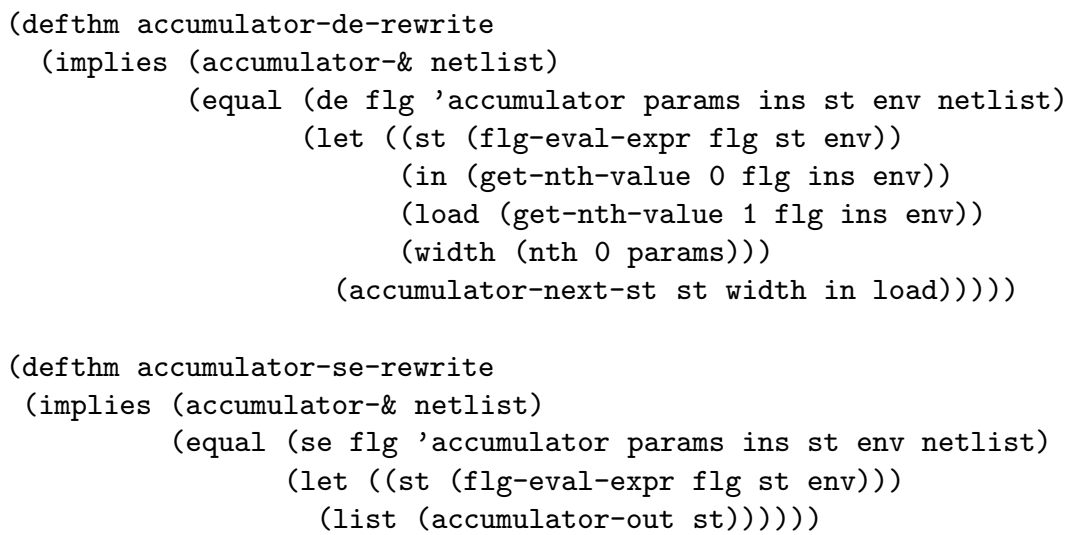

We now can prove properties about the ACL2 model using the ACL2 theorem prover. For example, consider the following theorem:

(thm

(let* ((state1 (accumulator-next-st state0 width A (LOAD))) 
(state2 (accumulator-next-st state1 width B (ADD))))

(equal (accumulator-out state2) (bv-adder width $a$ b))) )

In this theorem, state 1 is the state of our accumulator after an arbitrary LOAD instruction (i.e. the load input to the accumulator is high), and state2 is the state after following this LOAD with an ADD instruction (i.e. the load input is low). The theorem then states that the output of the accumulator is the addition of each cycles' inputs. We proved this theorem using the ACL2 theorem prover for any width accumulator. If we choose a specific width (e.g. a 32-bit accumulator), then this theorem can be proven automatically with our SAT-based decision procedure.

\subsection{Verifying Components of the TRIPS Processor}

We are using our verification system to verify components of the TRIPS processor. The TRIPS processor is a prototype next-generation processor being designed by a joint effort between the University of Texas and IBM [7. One novel aspect of the TRIPS microprocessor is that its memory is broken up into four pieces; each piece of memory has a separate cache and Load Store Queue (LSQ). We plan to verify the LSQ design, based on the design described in Sethumadhavan et al [6], using our verification system. We have already verified properties of its Data Status Network (DSN) component.

The DSN hardware provides the communication and buffering between four LSQ instances. Its design consists of 584 lines of Verilog code (including around 200 lines of comments), which we compile into a 427-line DE2 description (with no comments). We use our verifying compiler to translate this DE2 description into an ACL2 model and then prove the equivalence of the DE2 description and its ACL2 specification. Using a mixture of theorem proving and a SATbased decision procedure, we have proved properties that relate the output of the four DSN instances, communicating with each other over multiple cycles, to the output of a simplified machine; this simplified machine specifies the output that would be immediately produced if all communication were instantaneous.

\section{Conclusion}

The definition of the DE2 language provides a user with a hierarchical language for specifying FSMs. By deeply embedding the definition of DE2 within the ACL2 functional logic, we have provided a proof theory for verifying DE2 module descriptions with respect to a number of primitive interpretations. The extensible structure of the DE2 language and its general-purpose annotation language allow a user to embed other types of information, such as a module's size, specification, layout, power requirements, and signal types. Instead of just verifying large netlists, we often compare netlists or transform one netlist into another netlist in a provably correct manner. We have extended the ACL2 theorem-proving system with a SAT procedure that can provide counter examples. We also have proved the correctness of functions that automatically 
generate circuits; this can greatly reduce the amount of DE2 module definitions written by a user.

We believe that the design of DE2 more closely fulfills the needs of modern hardware design and specification than traditional HDLs. The increasing demands placed on hardware or FSM specification languages is presently being served by embedding all kinds of extra information in the form of comments into a traditional HDL. This process forces non-standard, non-portable use of HDLs, and prevents there from being a single design description that can be accessed by all pre- and post-silicon development tools. We believe that DE2 is the first formal attempt to integrate disparate design data into a single formalism. We believe future design systems should include similar features.

The DE2 language, annotation system, and semantics provide a user with a uniform means of specifying and verifying a wide variety of both functional and extrinsic properties. We continue to expand the size and type of designs that we have verified. In the future, we want to use DE2 to capture existing design elements to ease the reuse problem. Typically, in an industrial design flow, when a previously designed and verified design element is used in a new design, the verification has to be completely redone. Our ability to specify and verify modules in a hierarchical manner permits the reuse of prior verifications, and perhaps this verification reuse is the real key. Being able to reuse the design and the effort required to validate it will greatly reduce the effort of reusing previously designed modules.

\section{References}

1. Per Bjesse, Koen Claessen, Mary Sheeran, and Satnam Singh. Lava: Hardware Design in Haskell. The International Conference on Functional Programming (ICFP), pages 174-184, Volume 32, Number 1, ACM Press, 1998.

2. Mary Sheeran. Generating Fast Multipliers Using Clever Circuits. In Alan J. Hu and Andrew K. Martin, editors, Formal Methods in Computer-Aided Design (FM$C A D$ ), pages 6-20, LNCS, Volume 3312, Springer Verlag, 2004.

3. Sava Krstic and John Matthews. Semantics of the reFLect Language. Principles and Practice of Declarative Programming (PPDP), pages 32-42, ACM Press, 2004.

4. Mark D. Aagaard, Robert B. Jones, and Carl-Johan H. Seger. Lifted-FL: A Pragmatic Implementation of Combined Model Checking and Theorem Proving. Theorem Proving in Higher Order Logics (TPHOLs), LNCS, Volume 1690, Springer Verlag, 1999.

5. Mark D. Aagaard, Robert B. Jones, John W. O'Leary, Carl-Johan H. Seger, and Thomas F Melham. A methodology for large-scale hardware verification. In Warren A. Hunt, Jr. and Steve Johnson, editors, Formal Methods in Computer-Aided Design (FMCAD), LNCS, Volume 1954, Springer Verlag, 2000.

6. S. Sethumadhavan, R.Desikan, D.Burger, C.R.Moore and S.W.Keckler. Scalable Hardware Memory Disambiguation for High ILP Processors (Load/Store Queue Design). 36th International Symposium on Microarchitecture (MICRO 36), pages 399-410, 2003.

7. The Tera-op Reliable Intelligently adaptive Processing System(TRIPS), http://www.cs.utexas.edu/users/cart/trips/ 
8. Bishop Brock, Matt Kaufmann, and J Moore. ACL2 Theorems about Commercial Microprocessors. In M. Srivas and A. Camilleri, editors, Formal Methods in Computer-Aided Design (FMCAD'96), pages 275-293, LNCS, Volume 1166, Springer-Verlag, 1996.

9. Jun Sawada. Formal Verification of an Advanced Pipelined Machine. PhD Thesis, University of Texas at Austin, 1999.

10. Warren A. Hunt, Jr. The DE Language. Computer-aided Reasoning: ACL2 case studies, pages 151-166, Kluwer Academic Publishers, 2000.

11. Robert S. Boyer and J Strother Moore. A Computational Logic Handbook. Academic Press, Boston, 1988.

12. M. J. C. Gordon and T. F. Melham (editors). Introduction to HOL: A Theorem Proving Environment for Higher-Order Logic. Cambridge University Press, 1993.

13. Richard Boulton, Andrew Gordon, Mike Gordon, John Harrison, John Herbert, and John Van Tassel. Experience with Embedding Hardware Description Languages in HOL, Theorem Provers in Circuit Design, pages 129-156, IFIP Transactions A-10, Elsevier Science Publishers, 1992.

14. Mike Gordon. Why Higher-order Logic is a Good Formalism for Specifying and Verifying Hardware. Technical Report 77, University of Cambridge, Computer Laboratory, 1985.

15. Warren A. Hunt, Jr. and Bishop C. Brock. A Formal HDL and Its Use in the FM9001 Verification. In C.A.R. Hoare and M.J.C. Gordon, editors, Mechanized Reasoning and Hardware Design, pages 35-48, Prentice-Hall International Series in Computer Science, 1992.

16. Matt Kaufmann and J Strother Moore. ACL2: An Industrial Strength Version of NQTHM. Eleventh Annual Conference on Computer Assurance (COMPASS-96), pages 23-34, IEEE Computer Society Press, 1996.

17. Guy Steele. Common Lisp: The Lanugage, Second Edition. Digital Press, 1990.

18. Phillip J. Windley and Michael L. Coe. A Correctness Model for Pipelined Microprocessors, Theorem Provers in Circuit Design: Theory, Practice, and Experience, LNCS, Volume 901, Springer Verlag, pages 33-51, 1995. 